\title{
Féeries
}

Études sur le conte merveilleuX, XVII $-\mathrm{XIX}{ }^{\mathrm{e}}$ siècle

\section{Voyage aux sources des mille et une heures, contes péruviens}

\section{Marie-Françoise Bosquet et Régine Daoulas}

\section{(2) OpenEdition}

1 Journals

Édition électronique

URL : http://journals.openedition.org/feeries/167

DOI : 10.4000/feeries. 167

ISSN : 1957-7753

Éditeur

UGA Éditions/Université Grenoble Alpes

\section{Édition imprimée}

Date de publication : 1 février 2006

Pagination : 329-351

ISBN : 2-84310-082-8

ISSN : $1766-2842$

\section{Référence électronique}

Marie-Françoise Bosquet et Régine Daoulas, « Voyage aux sources des mille et une heures, contes péruviens », Féeries [En ligne], 3 | 2006, mis en ligne le 07 février 2007, consulté le 08 septembre 2020. URL : http://journals.openedition.org/feeries/167 ; DOI : https://doi.org/10.4000/feeries.167

Ce document a été généré automatiquement le 8 septembre 2020

(c) Féeries 


\title{
Voyage aux sources des mille et une heures, contes péruviens
}

\author{
Marie-Françoise Bosquet et Régine Daoulas
}

1 TOUTE UNE EFFLORESCENCE LITTÉRAIRE se structure autour du conte oriental depuis l'engouement suscité par la traduction par Galland des Mille et Une Nuits, et Les Mille et Une Heures, Contes péruviens participent de cette mode. Cette œuvre est généralement attribuée à Thomas-Simon Gueullette: en l'état actuel de nos recherches, seule la continuation du recueil qui s'interrompt brutalement à la LVIII ${ }^{\mathrm{e}}$ Heure dans l'édition de 1733 est assurément de la main de Gueullette puisqu'il l'a reconnue dans le codicille joint à son testament ${ }^{1}$. C'est en 1759 que paraît l'édition complète des Mille et Une Heures, Nouvelle édition, revue, corrigée \& considérablement augmentée par l'Auteur à laquelle nous nous référons ici².

2 Qu'il s'agisse d'une écriture à une main ou à deux mains, Gueullette a " revu » et " augmenté » l'édition de 1733 : enclin à satisfaire le goût du public et à se couler dans l'air du temps, il n'est guère singulier qu'il ait choisi d'écrire, ou de revoir et compléter, des contes situés dans une atmosphère péruvienne après avoir produit des contes tartares, chinois et mongols. D'autant moins singulier qu'il était un lecteur assidu, un voyageur en cabinet, féru d'un Orient qui englobait les Indes occidentales. La Route des Indes menait en Amérique où était entretenu un commerce privilégié avec sa partie la plus riche, le Mexique et surtout le Pérou dont les mines d'or et d'argent étaient devenues mythiques. Ainsi coexistent le titre, avec sa référence à l'Orient des Mille et Une Nuits, et le sous-titre de l'œuvre : les «Indes » incluaient le Pérou et le rattachaient à l'Orient dans l'esprit de l'époque. Gueullette, à la note 6 de la LXIV Heure, désigne le Mexique et le Pérou comme faisant partie des "Indes »; dans le conte-cadre et les abondantes notes de l'œuvre, la dénomination d' «Indiens » pour désigner les Péruviens est utilisée comme l'usage le voulait.

3 Mais il reste à comprendre pourquoi l'auteur a choisi d'installer son recueil, à l'opposé, à «l'envers » de l'Occident, dans un axe vertical Nord-Sud, et non plus horizontal Ouest-Est, dans un Nouveau Monde précolombien; et surtout à quelles sources il a puisé son inspiration et comment il les a utilisées. 
Le basculement du conte oriental selon un axe nord-sud

Une conjonction de circonstances historiques et scientifiques laisse entrevoir une explication plausible du changement d'axe du conte oriental: il découlerait d'une forme de synergie événementielle et scientifique.

5 Tout d'abord, rappelons qu'à ce moment de l'Histoire, la dynastie espagnole des Habsbourg a laissé place aux Bourbons et que le roi, après une longue guerre de succession et le Traité d'Utrecht en 1713 est Felipe Quinto de Borbón, Philippe V de Bourbon, duc d'Anjou, petit-fils de Louis XIV. Avec les Bourbons, l'influence française pénètre en Espagne, les relations entre les deux pays devenant plus harmonieuses.

6 Le Pérou est un Vice-Royaume de la couronne espagnole, Virreinato del Perú qui n'obtiendra son indépendance qu'en 1825. Le Potosí et ses fameuses mines d'argent citées dans l'épître introductive des Contes péruviens, font partie du Haut Pérou, province du Vice-Royaume.

7 Soulignons ensuite combien le Nouveau Monde et l'Amérique latine en particulier participent du goût des sciences commun aux salons. T.S. Gueullette (pour autant qu'on lui attribue l'ensemble des Contes péruviens) vivait d'ailleurs de cet esprit des Lumières ainsi qu'en témoigne J.E. Gueullette qui nous donne une idée de ses occupations littéraires abondantes, de ses curiosités érudites et de tous les lettrés et hommes talentueux qu'il fréquentait dans une vie bourgeoise bien remplie ${ }^{3}$. Du reste, une sorte de filiation semble s'être établie pour le lettré contemporain entre Voltaire, maître de la satire masquée en conte et Gueullette, si l'on considère le rapprochement que l'auteur de la notice sur Gueullette dans le Cabinet des Fées opère en 1786 entre Zadig et les Soirées bretonnes ${ }^{4}$ : Voltaire se serait " placé un moment dans la classe des imitateurs de Gueullette ${ }^{5}$ » ou à tout le moins « M. Gueullette a laissé des desseins, dont un grand maître a tiré parti ", affirme J.E. Gueullette ${ }^{6}$. Ainsi les idées circulent de l'un à l'autre ; et lorsque l'on sait que Voltaire peut être considéré comme à l'origine du voyage de La Condamine à l'équateur puisque c'est lui, ami personnel de La Condamine, qui a usé de toute son influence auprès de l'Académie des Sciences pour promouvoir ladite expédition en Amérique, on a peut être l'une des raisons, entre autres facteurs, qui a poussé l'auteur à installer le récit-cadre des Mille et Une Heures en Amérique : on peut y lire l'impact du projet de l'expédition de Charles de La Condamine, projet lui-même lié à la discussion véhémente opposant deux groupes de scientifiques.

8 En effet, l'un des grands problèmes scientifiques du moment était celui de la configuration exacte de la terre. Isaac Newton (1642-1727) avait émis l'hypothèse que la terre était un "ellipsoïde de révolution aplati », c'est-à-dire que le rayon équatorial devait être plus long que le rayon polaire. La théorie newtonienne, soutenue par Voltaire, était controversée par une dynastie d'astronomes, les Cassini. Irène Passeron, chercheur au CNRS, explicite :

Pourquoi cette variation de la gravité le long d'un méridien - ce grand cercle qui passe par les pôles - était-elle si importante? C'est que la nature de cette gravité, son mode d'action, étaient au centre des discussions théoriques de mécanique et de physique autour des années 1700 . C'est aussi que ces discussions interféraient avec des mesures astronomiques et cartographiques, les unes et les autres outils cruciaux dans le contrôle des mers et des terres. Enfin l'«opinion» des salons pouvait s'en approprier une facette, la divergence entre cartésiens et «newtoniens» sur la «figure de la terre ». [...] Une expédition lointaine financée par le Roi se justifiait: aux objectifs classiques, relevés géographiques, prélèvements botaniques, observations [...], pouvait s'en ajouter un qui 
contribuerait à la gloire du royaume : prouver une théorie à la portée universelle, celle de l'attraction universelle, justement ${ }^{7}$. expéditions: l'une en Laponie, avec pour chef d'expédition Maupertuis, l'autre à l'équateur, à la Audiencia de Quito, rattachée au vice-royaume du Pérou, sous la direction de Louis Godin, mathématicien, assisté de La Condamine. L'histoire ne retiendra que ce dernier nom. Cette dernière expédition, protégée par Maurepas ministre de la Marine, partit de La Rochelle, après avoir été longuement discutée et préparée en mai 1735, à l'époque où s'écrivaient les Contes péruviens. Le voyage de La Condamine en Amérique inaugure une nouvelle période de l'histoire des découvertes, les objectifs deviennent "scientifiques ", qu'il s'agisse de relevés, de prélèvements botaniques ou d'observations naturalistes ou anthropologiques : «Ce ne sont plus les conquistadors, les missionnaires ou les aventuriers qui occupent le devant de la scène dit Hélène Minguet dans sa préface au Voyage sur l'Amazone $e^{8}$ - le xviII ${ }^{e}$ siècle voit se développer un intérêt réellement scientifique pour le Nouveau Monde, comme d'ailleurs pour les autres parties connues ou peu connues ». Fait qu'avait déjà souligné A.D. Coderre : "Désormais nous aurons de vrais voyageurs qui s'occuperont de [...] connaître les coutumes sociales et politiques des nations d'Asie, leurs croyances et pratiques religieuses et le détail de leurs mœurs ", et, rajouterons-nous, d'en compiler leur relation dans des journaux de bord, véritable marqueterie de savoirs. Les esprits éclairés recommandaient aux voyageurs de savoir observer pour rapporter dans leur patrie une "moisson » de curiosités et de connaissances utiles. Or, par certains côtés le récit-cadre et les notes des Contes péruviens se rapprochent de la littérature de voyage, du moins d'un voyage dans le temps et en cabinet de lecture, ce que nous verrons plus avant.

Un certain nombre de dates sont là pour étayer notre hypothèse d'une synergie découlant d'un contexte historique et scientifique à l'origine des Contes péruviens :

11 1732-1734 - Maupertuis et Voltaire défendent les théories newtoniennes et proposent une vérification sur le terrain, à la Audiencia de Quito, rattachée au vice-royaume du Pérou.

1733 - première édition des Contes péruviens.

1735 - départ de l'expédition de La Condamine vers le Nouveau Monde ; 1743 - descente de l'Amazone.

1745 - Relation abrégée d'un voyage fait dans l'intérieur de l'Amérique méridionale, depuis la côte de la mer du Sud jusqu'aux côtes du Brésil et de la Guyane, en descendant la rivière des Amazones (Relation lue à l'assemblée publique de l'Académie des Sciences, le 28 avril 1745, par M. de La Condamine de la même Académie).

1759 - Nouvelle édition, revue, corrigée \& considérablement augmentée par l'Auteur. Dans son avis au lecteur, l'auteur des Contes péruviens imagine qu'un cahier a été trouvé à Blois, dans la vente des livres d'un ecclésiastique, portant pour titre «Suite et conclusions des C.P. ${ }^{9}$ " dans le goût du jour et des salons et de répondre aux Mille et Une Nuits orientales par Mille et Une Heures américano-péruviennes.

L'auteur s'assure ainsi du double intérêt d'un lectorat passionné de contes et épris d'exotisme, source de fabuleux. Gueullette affirmera d'ailleurs, dans sa brochure Les Nuits parisiennes: «Je travaille sans aucun intérêt mais seulement pour voir si je puis 
attraper le goût du public ${ }^{10}$ ». Or il sait que ce lectorat recherche la nouveauté et que le basculement à $90^{\circ}$ de l'axe oriental usuel Ouest-Est en axe Nord-Sud risque de plaire parce qu'original ${ }^{11}$ : les contes passent ainsi la fameuse ligne de l'équateur, ce qui, pour le XVIII ${ }^{\mathrm{e}}$ siècle a une importance symbolique. Ce passage revêtait le sens d'une initiation, marquée, sur les navires, par un baptême burlesque: les voyageurs s'apprêtaient à affronter un monde autre avec sa part de mystère. Les lecteurs de contes ont conscience, eux aussi, de pénétrer dans l'envers du monde et leur curiosité ne peut qu'être attisée.

D'ailleurs l'auteur revendique à l'initiale de son recueil, dans l'« Épître à $\mathrm{M}^{\mathrm{me}}$ Von der Stund ", « le mérite de la singularité » de ses Heures qui viennent d'au delà des «mers immenses » d'où l'on perd de vue «l'Étoile du Nord », c'est-à-dire tout repère habituel ; elles viennent des "extrémités de l'Univers ", de «l'autre hémisphère » comme pour « dés-orienter » le lecteur à l'instar des récits de voyage qui permettent la découverte d'un nouvel univers. Lui-même d'ailleurs se présente comme un voyageur égaré ; il était parti à la recherche de "certains mémoires » remplis de «merveilles du Nord»: "Je pris le parti de m'embarquer, ne comptant faire qu'une courte promenade » (p. III). Or il se retrouve aux antipodes: est-ce annoncer qu'il construira ses contes en bouleversant les habitudes littéraires du genre oriental désormais établi ? Il prévient immédiatement qu'il ne se donnera pas cette permission : l'origine de ses contes ne modifiera pas les règles du jeu orientalisant qu'il a déjà expérimentées, lui et «mille autres ». Il situe, non sans humour, son œuvre entre mentir-vrai et vraisemblance, à travers un raccourci saisissant du Nord européen au Sud américain : "N'allez pourtant pas vous imaginer, Madame, que j'ai été chercher dans l'autre hémisphère le privilège de débiter des fictions. Malgré le Proverbe, a beau mentir qui vient de loin, ce n'est plus la mode d'aller chercher ce droit au-delà des mers. Mille auteurs de ma connaissance l'ont acquis sans sortir de leur cabinet » (p. IV). Mille auteurs... mille ouvrages... mille nuits, mille jours, mille quart d'heures et enfin, Les Mille et Une Heures. Dans l'«Avertissement des Libraires », ce nouveau recueil est résolument classé aux côtés des œuvres orientales de l'axe Ouest-Est: «... nous avons cru pouvoir mettre sous presse un Livre, qui pouvoit figurer dans les Cabinets avec les Contes Arabes, Persans et Tartares. Il nous a paru que le dessein de l'auteur était le même » (p. VI-VII).

En gardant le modèle des Mille et Une Nuits, l'auteur ré-oriente le lecteur dés-orienté par le cadre péruvien: le but est ludique! Il s'agit, pour le conteur et son lecteur, de s'amuser dans le mouvement de bascule de l'axe oriental habituel jusqu'à perdre les repères usuels développés par la connaissance de ses civilisations. Le renouvellement de l'intérêt est ainsi assuré et l'idéal classique, instruire et amuser, respecté.

Aux sources des Mille et Une Heures

Que Gueullette soit ou non l'auteur de l'ensemble de l'œuvre ${ }^{12}$, la question est posée de savoir d'où proviennent les connaissances de l'auteur sur l'empire inca. Or nous allons démontrer que les Contes péruviens procèdent notamment d'une lecture attentive des Commentaires royaux (Comentarios Reales) de Garcilaso de la Vega, el Inca. Mais furent-ils lus en version originale ou en traduction? C'est ce que nous ne saurions dire.

Le conteur dispose de deux types de traduction en français : l'une, la plus ancienne, est celle de Jean Baudoin ${ }^{13}$ datant de 1633 ; l'autre, de Thomas François Dalibard ${ }^{14}$, n'a pu qu'inspirer, éventuellement, l'édition des Contes péruviens de 1759 car elle date de 1744. T.F. Dalibard signale dans sa Préface huit éditions de la traduction de J. Baudoin, ce qui constitue bien la preuve de l'intérêt ${ }^{15}$ que suscite l'ouvrage de Garcilaso qui est, par 
exemple, utilisé par $\mathrm{M}^{\mathrm{me}}$ de Graffigny pour ses Lettres péruviennes. Si J. Baudoin n'a " pour ainsi dire, fait qu'une Version littérale de cet Ouvrage » et s'il y a « laissé toute la confusion qui se trouvoit dans l'Original Espagnol ${ }^{16}$ ", Dalibard se targue, lui, d'avoir «tâché de remettre plus d'ordre dans les matières, sans rien retrancher de l'Original » car il estime que Garcilaso a fait "un ouvrage fort confus »: "Guerre, Politique, Religion, Sciences, Arts, Histoire naturelle, tout y est confondu ${ }^{17}$ ». Lorsqu'on compare ces deux traductions avec le texte des Contes péruviens, il apparaît que l'auteur s'est servi de J. Baudoin, à moins qu'il n'ait été capable de comprendre directement les Comentarios Reales.

Ce que nous pouvons affirmer sans conteste, c'est que le récit-cadre quasiment en son entier sort tout droit du tome II des Comentarios Reales, qu'il s'agisse des Vierges du Soleil : livre IV; de l'Inca Yahuar Huacac, toujours livre IV; ou de l'Inca Viracocha, livre V. De tels emprunts d'un auteur à l'autre faisaient partie de la coutume littéraire et T.S. Gueullette lui-même n'en a pas fait mystère pour ses contes orientaux avérés. $\mathrm{Ne}$ déclare-t-il pas dans l'« Avis au lecteur » des Sultanes de Guzarate, Contes mogols :

[...] plusieurs de nos auteurs romanciers n'ont pas dédaigné de puiser dans ces sources alors très peu connues, des histoires dont quelquefois même ils n'ont fait que changer les noms. Je ne dis pas ceci pour le leur reprocher; au contraire j'estime que nous leur avons beaucoup d'obligation d'avoir tiré, pour ainsi dire, ces ouvrages de l'obscurité. À leur exemple, si l'on reconnaît quelque fonds de mes histoires, je crois que l'on aura autant d'indulgence pour moi que l'on en a eu pour ces messieurs.

L'« Avertissement des Libraires » des Contes péruviens commence par poser la question de «l'origine des Contes » en usant de la prétérition et d'une énumération hasardeuse, comme pour brouiller la piste: «Nous n'entreprendrons pas d'informer le public de l'origine des Contes que nous publions : s'ils ont été trouvés dans quelque caverne de l'Amérique, dans les ruines de quelque vieux palais des Incas, ou parmi les effets de quelque Espagnol descendu des conquérans du Pérou » (p. V). C'est bien Garcilaso de la Vega el Inca qui vient d'être désigné !

20 Garcilaso de la Vega - né à Cusco en 1539 et mort à Córdoba 1616 - est le nom qu'adopta en 1563 Gómez Suárez de Figueroa, fils du conquistador espagnol Sebastián Garcilaso de la Vega et d'une princesse inca, Isabel Chimpu Ocllo Xuarez, cousine germaine du dernier empereur inca, Atahuallpa. En 1560, il partit pour l'Espagne où il suivit une carrière militaire. À partir de 1590 , il se consacra à un travail d'historien et d'humaniste, alliant à ses souvenirs une relecture critique des autres chroniqueurs, en particulier l'œuvre du Père Valera. Garcilaso est le premier grand prosateur hispanoaméricain, auteur d'ouvrages historiques : La Florida del Inca, 1605 - Comentarios Reales, 1609 - Historia general del Perú, 1617.

21 Sa double appartenance et son désir d'intégration vont le mener vers une représentation singulière du passé incasique dans une tentative de christianiser les mythes fondateurs incas. Les Incas ne possédaient pas d'écriture, tout était transmis oralement et les transcriptions pouvaient, en conséquence, être entachées par des idées pré-construites ou par des représentations européocentristes. Pour Garcilaso de la Vega, el Inca, il s'agit en outre d'exalter sa double filiation inca et espagnole, dominée/ dominante, tout en ménageant la susceptibilité exacerbée et tyrannique du Saint Tribunal de l'Inquisition. Les éditions Mercurio précisent dans leur prologue au tome II : « Les Commentaires Royaux furent publiés il y a environ trois cent soixante-dix ans mais leur édition et distribution furent interdites pendant deux cents ans sur ordre 
du Conseil des Indes et de l'Inquisition ${ }^{18}$ ». Mise à l'index due sans nul doute à la philosophie très personnelle de Garcilaso, ce que l'historien cubain Raimundo Lazo ${ }^{19}$ souligne dans son ouvrage Historia de la literatura Hispanoamerina. Il s'agit d'une philosophie hétérogène qui mêle des éléments chrétiens et néo-platoniciens, aussi bien à la pensée de Sénèque qu'au subconscient inca. Il en résulte, selon Lazo, une fusion de l'histoire et du mythe, dans l'équivalence de sens et de valeur que leur donnait Garcilaso ; mais toujours selon le critique, il ne faut pas oublier la double perspective des Commentaires, historique et personnelle ; l'histoire est davantage représentation que reconstruction. Quant à l'évocation intime d'un Pérou natal, elle est nécessairement nostalgique car liée à la jeunesse du métis bâtard et en quelque sorte exilé. Lazo insiste également sur la façon singulière qu'avait Garcilaso d'ordonner les matériaux historiques et fabuleux dans la première partie des Commentaires pour éviter la monotonie : Garcilaso entrelace librement histoire interne, la culture et la civilisation indigène, et histoire externe, politique et militaire.

Les Commentaires royaux forment donc des chroniques et c'est sur cette liberté de traitement que va jouer l'auteur des Contes péruviens. Bernard Lavallé, dans L'Amérique espagnole de Colomb à Bolivar, définit ainsi les chroniques :

Dès la Découverte, puis la Conquête, le fait américain suscita une abondante littérature de souvenirs, de voyages, d'exposés divers, connus sous l'appellation globale et imprécise de "chroniques». D'intérêt très variable selon les auteurs, l'acuité et la perspicacité de leur regard, la richesse de leur expérience et le sens qu'ils donnaient à leur œuvre, ces chroniques constituèrent le premier pont entre les deux mondes, dans la mesure où, pratiquement, elles furent longtemps le seul moyen de faire connaître le Nouveau à l'Ancien ${ }^{20}$.

Les chroniques de Garcilaso ont servi de matrice à l'imagination du conteur et ont tissé la trame des Contes péruviens tel un axe syntagmatique ou axe horizontal tandis que les contes adventices enchâssés formeraient l'axe ou les axes paradigmatiques. L'axe syntagmatique rapproche le recueil de la littérature de voyage en provoquant l'immersion dans l'univers incasique de référence - y compris grâce aux notes explicatives - et il permet l'enchaînement des maillons-contes ; l'axe paradigmatique, constitué par les contes, provoque, lui, l'immersion dans le domaine du merveilleux. La touche d'exotisme américain tend à disparaître dans cet axe et seules quelques notations géographiques en témoignent; encore bien souvent, l'auteur se contente-t-il d'accoler «suyu » à des noms de territoires imaginaires en rappel d'un motif exotique inca. De cette façon, il les authentifie ou du moins leur donne une coloration américaine. Le rôle charnière de l'axe syntagmatique contribue à installer cette fonction du mentir-vrai dans l'organisation narrative tout en établissant déjà une jonction avec le merveilleux par le vecteur de l'exotisme incasique.

Le travail de réécriture des sources dans le conte-cadre

24 Nous nous appuierons essentiellement sur le conte-cadre pour donner une idée de l'utilisation par le conteur des Commentaires Royaux, puisqu'il en porte la plus forte trace. Pour ce faire, nous suivrons l'ordre du récit de T.S. Gueullette et nous étaierons notre commentaire par les précisions scientifiques d'Alfred Métraux ${ }^{21}$.

1. La conduite du récit dans le conte-cadre et sa double coloration orientale - Orient des Mille et Une Nuits et "Orient» incaïque - sont elles-mêmes significatives de la manière dont l'auteur des Contes péruviens mêle ses deux sources: les chroniques du Pérou et Les Mille et Une Nuits. Le récit-cadre s'articule en quatre séquences à l'image des Mille et Un Quarts d'Heure ${ }^{22}$. 
sequence initiale instaure le cadre précolombien incaïque, la naissance mythique de «l'Empire de légende» et le choix d'une focalisation sur l'un des Incas, Yahuar Huacac et son fils Viracocha (fin $\mathrm{du} \mathrm{xv}^{\mathrm{e}}$ siècle). Toujours parce que les Incas ne connaissent pas l'écriture, la tradition orale permet l'intromission du fabuleux dans l'Histoire : conquêtes, déboires familiaux, songe prémonitoire et mythe de Viracocha (principale divinité du panthéon inca, créateur de toutes choses), s'entrecroisent tissant le «mentir-vrai» de cette séquence-prologue. Viracocha prend le pouvoir et démet son père Yahuar Huacac, lequel sombre dans une dépression suicidaire.

La deuxième séquence introduit le lecteur dans la Maison des femmes choisies ${ }^{23}$, Acllahuaci, vierges du soleil. Là encore, l'auteur des Contes péruviens a puisé chez Garcilaso ou chez Baudoin. Paramètre essentiel de la religion inca, les vierges du Soleil sont à la fois le motif exotique qui capte l'attention et le rouage premier de l'enchâssement des contes. Le conteur puise dans cette institution son inspiration pour créer le personnage dont la voix structure l'ensemble du recueil, Acllahua. Il en fait l'élue des dieux, chargée de la mission de sauver l'Inca et la renommée de la dynastie par son art de conter. De plus, il insère une note instructive sur l'organisation des vierges du soleil (tome I, p. 60 et 67) car il pressent toute la curiosité qu'elle soulève. Pourtant il ne s'étend pas sur la question comme Garcilaso qui, aux chapitres 1 et 2 du livre IV, insiste (comme Baudoin) sur l'importance des vierges, les comparant implicitement aux vierges romaines, dans leur fonction, dans le châtiment prévu s'il y a faute - elles étaient enterrées vivantes - et dans le choix de la traduction du terme qualifiant la Supérieure des Maisons du Soleil: mamacuna, «matrone». La représentation du conteur participe non seulement de la vestale romaine, mais aussi de la religieuse chrétienne: la vestale romaine était choisie parmi les familles patriciennes, mais n'était pas enfermée comme la vierge du soleil, elle exerçait son ministère pendant trente ans et pouvait ensuite se marier; les religieuses étaient mariées spirituellement à Dieu par l'intermédiaire de l'Église, et certaines vivaient dans une clôture totale. Dans les Contes péruviens, à propos des vierges du Soleil, affleurent très nettement, avec un degré de plus par rapport aux Commentaires royaux, les deux strates des cultures romaine et chrétienne qui se superposent à la culture inca.

troisième séquence s'inspire directement du conte-cadre des Mille et Une Nuits ou des Mille et Un Quarts d'Heure : l'axe oriental Ouest-Est est ainsi fermement rétabli bien qu'il n'ait jamais été absent des précédentes séquences. Par contes interposés s'engage une plaidoirie qu'Allahua remporte : elle obtient de la mamacuna l'autorisation d'accomplir sa mission auprès de l'Inca. Cependant le contexte inca, qui situe la scène de contage dans la Maison des vierges choisies, a toujours pour source les Commentaires royaux.

La quatrième séquence, puise, comme la précédente, ses racines à la fois dans Les Mille et Une Nuits - Acllahua s'offre de prouver à l'Inca, par des exemples contés, que des princes, connaissant des situations plus tragiques que la sienne, ont trouvé le courage de les surpasser, défi qui n'est pas sans analogie avec celui lancé par Schahzenan à son frère Schahriar ${ }^{24}$ - et dans l'histoire de l'Inca Yahuar Huacac dépossédé de son empire par son fils Viracocha.

Ainsi le récit-cadre emprunte certains de ses éléments structurants à la tradition des contes orientaux issus de la traduction de Galland; surtout, il synthétise les premiers livres (1 à 5 essentiellement) des Commentaires Royaux de Garcilaso de la Vega, en opérant des choix, en évitant les longueurs didactiques et en ciblant les épisodes les plus fabuleux. Faut-il souligner que la critique historique place les Commentaires dans le 
registre de l'épopée plus que dans celui de l'Histoire sans néanmoins nier l'importance de la documentation amassée ? Le conteur puise, selon son besoin original, dans les Commentaires et compose un "patchwork », technique qui renvoie à la marqueterie des relations de voyage où la ré-écriture implique un modelage des données brutes.

2. Les prolégomènes du récit-cadre démontrent la même méconnaissance des cultures pré-incasiques aussi bien chez l'auteur des Contes péruviens que chez les chroniqueurs espagnols, Cieza de León, Vaca de Castro, Juan de Betanzos, Diego de Ortega y Morejón, et peruano-espagnols, le Père Blas Valera ${ }^{25}$ et Garcilaso de la Vega el Inca, parce que les chroniques installent en maintes occasions la glorification de la civilisation inca et de son caractère fabuleux à partir du rabaissement des cultures précédentes ravalées au rang de cavernicoles, esprit que l'on retrouve dans les premières pages des Contes péruviens.

32 En effet, les deux premières pages sont la traduction quasi littérale (seconde colonne) d'un paragraphe du chapitre 15 du livre I des Comentarios Reales ${ }^{26}$ (première colonne), ou bien la reprise de la traduction de J. Baudoin au livre I, chapitre $\mathrm{xV}^{27}$ (troisième colonne) :

Ce tableau, qui place au croisement de deux sources similaires le texte des Contes péruviens, permet de comprendre notre incertitude quant à son origine exacte, texte original ou traduction. Il serait très vraisemblable d'ailleurs que le conteur ait eu à sa disposition les deux textes car il est remarquable de noter qu'il n'a pratiquement pas puisé sa matière dans les passages des chroniques où Baudoin s'écarte quelque peu de Garcilaso.

Pour donner quelques exemples des ignorances rencontrées tant chez le conteur que chez les chroniqueurs touchant les cultures pré-incasiques, nous citerons celle de Chavín - qui s'est étendue sur la côte Nord jusqu'au lac de Titicaca de 1800 à 200 av. J.C. et qui introduisit la culture du maïs, céréale vitale; les cultures Mochica et Nazca, dites de la période classique, entre 200 et 1100 après J.-C. qui formèrent des États très organisés militairement et de plus en plus stratifiés socialement au fur et à mesure que progressaient la technique agricole et la maitrise de l'eau; cultures remarquables également par leur architecture, leur orfèvrerie, leur céramique ; enfin la civilisation Chimú, post-classique, héritière de la culture Mochica, qui connut un développement harmonieux pendant quasiment cinq siècles (1000-1470) jusqu'à sa conquête par les Incas. La méconnaissance de toutes ces cultures signe bien l'origine d'où sont issus les Contes péruviens à savoir Garcilaso qui lui-même entérine la tradition des chroniqueurs espagnols.

35 3. La manière synthétique dont le conteur utilise Garcilaso et l'élagage qu'il pratique apparaissent nettement lorsque les premières pages des Contes péruviens expliquent l'origine mythique et l'organisation théocratique de l'empire inca.

Le conteur ne développe pas, comme dans les Commentaires royaux, les trois mythes qui alimentent l'histoire de l'apparition des Incas. Puisque tous trois reconnaissent Manco Capac comme fondateur de l'empire inca, il choisit le plus merveilleux et le plus simple, le premier. Il satisfait ainsi le goût du lecteur à s'instruire, sans nuire par la longueur au plaisir du conte qui se veut rapide. Ce faisant, il représente assez fidèlement une des sources de la mythologie inca et de la naissance de l'empire de légende (1200-1532). Les trois premières pages, notes incluses, forment une synthèse de ce premier mythe situé dans le chapitre 15, livre I, des Comentarios ${ }^{28}$ : le Soleil, dieu suprême, tire des eaux du 
Lac Titicaca ses deux enfants Manco Cápac et Mama Ocllo ; il leur confie son bâton d'or en ordonnant qu'une ville soit fondée là où ce bâton, lancé vers le sol, resterait planté ; le bâton s'enfonce dans la terre fertile de la vallée de Cusco. L'auteur des Contes péruviens ne garde que le nom des fondateurs en soulignant leur mariage quoique frère et sœur, et les instructions décisives pour le progrès des "nations infortunées » (p. 2) qu'ils civilisent. Par cet art de la condensation, il évite d'avoir à trancher parmi les différences qu'il peut éventuellement relever entre Garcilaso et son traducteur, J. Baudoin: certains détails diffèrent, par exemple à propos du bâton qui fixe la fondation de la ville ${ }^{29}$, ou bien des permutations d'éléments s'opèrent entre la deuxième et la troisième légende ${ }^{30}$, plus complexes car mettant en scène quatre frères en quête d'une terre promise après un déluge. Le conteur ne signale pas la division en quatre régions de l'empire inca ou Tahuantinsuyu (Tahuantinsuyo) qui s'étendait sur les quatre aires de vent ou les quatre points cardinaux définissant quatre régions administratives ou suyu (suyo). Le Chinchaysuyu comprenait le Nord et le centre de la cordillère des Andes, le Continsuyu, (de l'ouest de Cusco au Pacifique), le Collasuyu, le Sud et l'altiplano, (Bolivie, Nord du Chili) l'Antisuyu, le Nord et le Nord-est jusqu'à l'Amazonie (Équateur, Sud de la Colombie, Pérou amazonien).

Les Contes péruviens rendent compte, toujours de façon synthétique par comparaison avec leur source, aux pages 4 et 5, d'une administration théocratique rigide et hiérarchisée qui réglait la vie de l'individu de sa naissance à sa mort. Ils ne s'étendent pas comme les Commentaires ${ }^{31}$ ou leur traducteur sur la puissance des Incas qui reposait sur le travail et l'union des peuples, l'empire étant une mosaïque de nations et de tribus. Ils ne précisent pas que la terre appartenait à l'Inca (terrestre, Sapa Inca) représentant du Soleil (Inca céleste, Inti) qui la distribuait aux paysans la cultivant. C'est le principe de l'ayllu, communauté rurale, véritable cellule de base de l'empire ; cellule sur laquelle le conteur fait l'impasse alors que l'aspect collectiviste qu'elle impliquait a intéressé au plus haut point l'abbé Raynal, Marmontel et surtout Morelly en 1753 dans sa Basiliade. Ce qui attire l'auteur des Contes est ce qui nourrit le récitcadre impérial et mythique, non l'utopisme qui peut s'en dégager. Il se contente de noter : «[...] l'empire des Yncas prit un tel accroissement, qu'il parvint à ce haut point de grandeur \& de puissance, où les historiens nous le dépeignent » (p. 4).

Il note ainsi en passant et comme sans y prendre garde - la synthèse est à tout le moins étonnante comparativement au texte de référence ${ }^{32}$ - un des deux paramètres essentiels unificateurs de cet empire de 1,7 millions de $\mathrm{km}^{2}$ : la religion, le second paramètre étant la langue dont il ne fait pas mention explicitement: «Le Soleil leur père leur avait enjoint à l'un \& à l'autre, d'instruire les peuples dans la religion qu'ils devaient professer, de leur prescrire les lois qu'ils devaient suivre $[. . .]^{33} »$. Or la religion est l'un des points qui retient le plus l'attention de Garcilaso tant il a le souci de dégager les liens qui peuvent rattacher le culte solaire des Incas au christianisme. Mais en même temps il note les deux difficultés majeures que les chroniqueurs espagnols rencontrèrent pour comprendre et retracer l'histoire de la dynastie inca et de la constitution de leur empire, c'est-à-dire le décalage entre leur civilisation chrétienne et celle des Incas, accentué par la difficulté à se comprendre d'une langue à l'autre :

La manière qu'avaient nos Espagnols d'écrire leurs histoires était d'interroger les Indiens en castillan sur ce qu'ils voulaient savoir. [...] mais comment ces barbares (les Indiens) plongés dans de profondes ténèbres pouvaient-ils comprendre le mystère de l'Incarnation ou celui de la Sainte Trinité. [...] Le pire était que chacun 
savait très peu de choses du langage de l'autre car la langue indienne est très difficile et les Indiens savaient fort peu de castillan à l'époque [...]. ${ }^{34}$ commirent-ils de nombreuses erreurs dans leurs chroniques: ils confondirent, par exemple, Pachacamac avec Rimac: Pachacamac, el Hacedor, est celui qui crée et celui qui soutient l'univers quand Rímac est celui qui parle. Les Contes péruviens depuis le récit-cadre jusqu'à l'Heure LVIII entérinent cette confusion: Rimac y est présenté comme le dieu suprême, oblitérant Pachacamac qui n'apparaît qu'à la fin de l'Heure LVIII, c'est-à-dire à la fin de l'édition de 1733. Il semble donc que l'auteur des Contes péruviens se soit d'abord fié aux chroniqueurs espagnols avant de rectifier sa position, sans doute après lecture ou relecture des chapitres 1 à 9 du livre II, tome II et du chapitre 30 du livre VI, tome II (p. 170-171) des Commentaires royaux. Il introduit alors Pachacamac à la dernière page de l'édition de 1733, en précisant à l'Heure LIX, dans l'édition de 1759, sa suprématie : «... il [Rimac] est inférieur au grand Pachacamac, créateur de l'univers» (p. 380). La "Conclusion» du récit-cadre commence d'ailleurs par une mise au point du panthéon inca, comme si l'auteur voulait définitivement effacer l'erreur commise : " [...] j'ai été avec plaisir, dit l'Inca à Acllahua, instruit du pouvoir des deux divinités dont je n'avois que des connoissances très superficielles » (p. 477-478).

Cette correction que les Contes péruviens apportent sur l'organisation divine inca, est sans doute révélatrice des lectures sur lesquelles leur écriture s'appuie : les œuvres des chroniqueurs espagnols sont issues des récits que transmettait la tradition orale; elles en sont sorties aussi riches qu'emplies de contradictions, aussi fabuleuses que déformées, car amplifiées à outrance et emplies d'erreurs. Garcilaso a lui-même parfaitement conscience des fluctuations de la tradition. Il qualifie l'histoire de l'origine des Incas de "fabulosa », c'est-à-dire à la fois fabuleuse, mythique et incroyable ${ }^{36}$, ce qui s'accorde directement avec l'écriture de contes!

41 4. Le conteur se sert donc des Commentaires royaux (livre IV, chapitres 20 à 24 et livre V, chapitres 17 à 22) pour installer son récit-cadre à la fois dans le domaine de l'Histoire et dans celui du merveilleux, car le choix de Yahuar Huacac et de son fils Viracocha n'est absolument pas anodin : ce sont, en effet, ceux qui condensent le mieux la chronique historique et la naissance du mythe. Le conteur se sent alors autorisé à reprendre directement le texte du chroniqueur.

Yahuar Huacac est probablement ${ }^{37}$ le septième Inca, fils de Inca Roca. Selon Vaca de Castro $^{38}$, Inca Roca mena une longue guerre contre un chef ennemi Tocay; les hommes de ce dernier s'emparèrent du fils d'Inca Roca mais au moment où ils allaient le tuer, l'enfant se mit à pleurer des larmes de sang, laissant ses bourreaux terrifiés. Après ce prodige, le fils du Soleil prit le nom de Yahuar Huacac, qui veut dire en quechua « qui pleure le sang ». Il régna une quarantaine d'années à Cusco et il eut de Mama Chizquia qui n'apparait pas chez Gueullette - six fils dont le premier deviendra le grand Viracocha qui vécut autour de 1430.

43

Comme l'indiquent fort exactement les Contes péruviens ${ }^{39}$, reproduisant fidèlement Garcilaso $^{40}$, à dix-neuf ans Viracocha était un jeune homme si rebelle et indiscipliné que son père Yahuar Huacac l'envoya garder des troupeaux loin de Cusco, sur les pâturages de Chita. A partir de là, le conteur fait œuvre de création romanesque en s'appuyant sur l'institution des Vierges choisies : il invente un prince rebelle et pervers au point d'enfreindre l'interdit qui préservait la chasteté des Vierges, alors que 
Garcilaso affirme : «C'était la loi, mais elle ne fut jamais appliquée parce que jamais l'on n'apprit que quelqu'un l'avait transgressée ${ }^{41} »$. De l'œuvre du chroniqueur à celle du conteur, la différence s'accentue: le conteur marie les ingrédients aptes à frapper l'imagination du lecteur et il approfondit par petites touches le caractère des protagonistes, montrant ici la faiblesse d'un Prince ne sachant dominer ses instincts et celle de Yahuar Huaca ne punissant pas son fils de mort, mais l'exilant simplement. L'épaisseur humaine démythifie certes le conte, mais elle le vivifie.

Les Contes péruviens empruntent aux Commentaires royaux l'épisode du songe prémonitoire : le Prince retourne au palais pour prévenir son père de la révolte des peuples Chancas dans le Chinchasuyu; un songe, qui prévoyait la victoire inca, l'en avait averti. Tout l'épisode guerrier qui s'ensuit devient une véritable geste portant la marque de la tonalité épique des Commentaires (chap. 24 du livre IV).

Le trait psychologique qui définit Yahuar Huacac (sa faiblesse), mais qui établit dans la même phrase la stratégie Chanca (p. 31) vient aussi de la plume de Garcilaso : "Les Chancas avaient remarqué que l'Inca Yahuar Huaca était peu belliqueux, qu'il était même pusillanime et angoissé par le mauvais présage que renfermait son nom. De surcroît ils le savaient très embarrassé par la mauvaise conduite de son fils ${ }^{42}$ ».

Cette fidélité à leur source entraîne sans doute ici les Contes vers un manque de vraisemblance : le Prince exhorte son père qui s'est enfui lâchement de sa capitale, à protéger Cusco parce que la chasteté des Vierges du Soleil risque d'être profanée par les ennemis! Ce changement radical d'attitude de la part d'un Prince oublieux du fait qu'il a été le premier et le seul jusqu'alors à avoir bafoué la règle de chasteté, a presque un effet comique sur le lecteur! Le discours de Viracocha à l'Inca est rapporté avec exactitude depuis sa source: le texte de Garcilaso est repris jusqu'aux points d'interrogation rhétoriques. Faudrait-il y voir un trait d'humour qui établirait implicitement une distance critique ou une trop grande fidélité qui dessert momentanément la vraisemblance?

Quoi qu'il en soit, le récit se fait héroïque; le conteur continue de s'inspirer directement du chroniqueur: seul et simplement armé d'une lance, Viracocha part défendre le Cusco, construisant sa stature de stratège et guerrier. L'un et l'autre utilisent le détail vrai qui colore le texte : les armées incas utilisaient des instruments à vent pour dynamiser le combat. Voici ce qu'écrit Garcilaso, livre V, chapitre $18:$ : [...] criant et vociférant, aux sons des trompettes et des timbales, des trompes et des conques, ils avancèrent les uns vers les autres ${ }^{43}$ ». Et en voici l'utilisation par le conteur (p. 44-45) : «[...] accompagné du son des haut-bois, des cornets, des atabales et des trompettes, l'Ynca Viracocha... ", qui ajoute une note savante sur l'utilisation des instruments de musique par les «Indiens » au combat.

Mais le conteur peut très explicitement souligner le merveilleux du texte : «Les Yncas étaient habiles à illustrer leurs avantures par des circonstances merveilleuses... » (note de la p. 46). Quand s'engagea la bataille décisive - disent les chroniques de Vaca de Castro à Garcilaso de la Vega - arrivèrent des bois environnants, des fidèles de l'Inca qui avaient appris la résistance de Viracocha. Parmi les combattants se répandit la nouvelle que c'étaient les rochers de la forêt qui se transformaient en hommes armés, sur l'ordre du Dieu Viracocha. La panique s'empara des Chancas qui se rendirent par milliers. De la même façon, le conteur rend compte de cette victoire inattendue et de cette légende d'une armée miraculeuse que le Créateur aurait suscitée du sol. Il se plaît à insister sur l'habillage merveilleux de la donnée historique ${ }^{44}$, peut-être pour 
authentifier cette partie du récit vis-à-vis du lecteur, par rapport aux éléments indubitablement magiques qui vont suivre; peut-être aussi, si l'on reprend la théorie de Catherine Langle ${ }^{45}$, pour avertir le lecteur et le prévenir contre « une adhésion sans recul au faux merveilleux ou à l'idéalisme romanesque "; sans doute enfin, parce que Garcilaso, dans ce chapitre $18 \mathrm{du}$ livre $\mathrm{V}$ et à propos de cette bataille décisive pour l'empire inca, s'appuie sur les pères Gerónimo Román (livre II de República de las Indias Occidentales) et Acosta qui nomment Viracocha "Seigneur universel et créateur de tout, ayant fait le ciel et le Soleil, le monde et les hommes » dans un parallèle singulier de « leur » Dieu chrétien et de ses miracles.

L'auteur des Contes péruviens suit encore le livre V de Garcilaso au chapitre 19 cette foisci, intitulé: "La générosité du prince Inca Viracocha après sa victoire ${ }^{46}$ ", en soulignant la grandeur d'âme de l'Inca après son triomphe. Cette transformation d'un prince irresponsable et rebelle en héros n'appartient pas à la création romanesque, mais au style complexe de la chronique. De même le conteur se contente-t-il de rendre littéralement le texte des chroniques lorsque Viracocha s'empare de l'insigne impérial : "Viracocha quitta la bordure jaune et en prit une rouge», "dejó la borla amarilla y tomó la colorada ${ }^{47} »$ : le mot «bordure » n'est pas approprié. A. Métraux parle d'une frange: "Le symbole de la dignité impériale était le llautu, tresse de différentes couleurs faisant cinq ou six fois le tour de la tête et retenant sur le front une frange en laine, la maskapaicha, dont chaque élément passait dans un tube d'or ${ }^{48}$ ". C'était la frange impériale. Le jaune était la couleur princière, le rouge, la couleur de l'Inca. Et non seulement Viracocha s'empara de l'insigne llautu, mais aussi de l'empire tout entier : il détrôna son père.

5. C'est encore chez Garcilaso que le conteur puise pour ciseler un des motifs enclenchant le dispositif des Heures : le désir de L'Inca de se suicider en buvant un poison.

Garcilaso avait signalé dès le chapitre 14 du livre I que le poison était utilisé pour tuer rapidement ou lentement, pour hébéter et priver de jugement, pour enlaidir les visages et les corps. Ce chapitre 14 indique, entre autres, les coutumes licencieuses des tribus barbares pré-incasiques, coutumes qui sont notées par le conteur au bas de la page 59 et seront reprises dans certaines Heures.

Garcilaso renoue avec ce thème au chapitre 20 du livre $V$ pour indiquer très brièvement, sinon l'état d'esprit de l'empereur dépossédé, Yahuar Huaca, du moins celui que lui supposaient les gens de son entourage ou peut-être même la représentation qu'il s'en faisait : «... d'autres disaient que l'infortuné Inca n'avait pas choisi la solution du poison [...]. D'autres, qui se disaient religieux, et renchérissaient sur la noblesse et la générosité de leurs Incas faisaient remarquer que l'Inca n'utiliserait pas le poison parce que c'était chose indigne des Incas fils du Soleil. D'autres disaient encore... ${ }^{49}$ ».

53 Le conteur saisit cette idée pour la prolonger. Il façonne son personnage : il a annoncé par petites touches impressionnistes la fragilité psychologique de Yahuar Huaca, son côté pleutre et hésitant qui l'oppose à la lignée conquérante qui l'a engendré. Il va, dans la séquence suivante, exposer avec finesse et sobriété la mélancolie désespérée de l'empereur dépossédé ${ }^{50}$. Aussi, quand Garcilaso clôt de façon tranchante l'épisode de Yahuar Huaca: "Y con esto dejaremos al Inca, llora sangre, para no hablar más de él », «Et sur ce, nous laisserons l'Inca, pleure du sang, et nous ne parlerons plus de lui », le conteur prend ici la relève et souligne le désespoir de l'empereur que sa fierté même 
accule au suicide, enclenchant, dans le même temps, ce que nous avons nommé la deuxième séquence du récit-cadre: la situation qui va générer les Heures s'intègre progressivement au récit-cadre.

Mais le personnage qui enclenche définitivement la succession des Heures est, nous l'avons déjà précisé, de l'invention du conteur, bien que partiellement calqué sur Schéhérazade. Il est cependant placé dans un cadre que l'auteur des Contes a pris chez Garcilaso: le cadre mythico-réel du Pavillon de la Lune où se trouve transportée Acllahua par une main lumineuse.

L'intrusion du merveilleux coïncide, ici encore, avec un cadre fabuleux, selon les dires de Garcilaso qui insiste surtout sur le Temple du Soleil. Certains critiques se demandent d'ailleurs jusqu'à quel point il est crédible, le Temple du Soleil ayant été presque totalement détruit. Le chapitre 21 du livre III de Garcilaso en son entier convoque l'idée d'une magnificence extraordinaire, y compris pour le Pavillon de la Lune.

Sur toute la hauteur [du Temple du Soleil] s'étendait une frise d'or très pur, large de deux paumes, qui l'entourait comme une couronne dorée [...]. Tout autour de ce cloitre s'ouvraient cinq très grandes salles carrées [...], la première salle était dédiée à la vénération de la Lune, sœur et épouse et par conséquent la plus proche du lieu d'adoration du Soleil. Tout, dans cette salle, était d'argent très pur: les murs, le toit ${ }^{51}$, les portes, les parois, afin que chacun pût se rendre compte, à la clarté splendide de l'argent, qu'il se trouvait dans la maison de la Lune $[. . .]^{52}$.

Le conteur n'utilise que ce pavillon comme décor à la vision d'Acllahua, mais de façon beaucoup plus sobre, l'action l'emportant sur la description: «Il [le bras magique lumineux] m'a transportée dans le pavillon de la lune et m'a portée sur un trône d'argent [...], mes yeux ont été éblouis de la lumière qui environnait le visage de la Lune, \& cette lumière glorieuse a fait une si vive impression que j'ai perdu connaissance quelque tems » (p. 64). Mais il se sert de la description des momies des reines incas dans sa note explicative : «Dans le pavillon de la lune, aux deux côtés de la figure, étaient les corps des reines décédées, rangés en ordre selon leur ancienneté » (p.64), et alors il suit de très près le chapitre 21 (livre III) de Garcilaso : «[En el aposento de la Luna] a un lado y otro de la imagén de la Luna estaban los cuerpos de las reinas difuntas por su orden y antigüedad ». Ce faisant, il installe encore une fois le détail «vrai » qui sert l'habillage exotique.

6. Il reste maintenant - car il serait inutile de passer en revue plus longuement les emprunts aux chroniques - à examiner brièvement l'utilisation des sources dans les notes du récit-cadre.

La première séquence du conte-cadre comporte un nombre important de notes de type didactique; elles sortent en droite ligne des Commentaires de Garcilaso, des premiers chapitres du livre I en ce qui concerne l'origine mythique des Incas, des premiers chapitres du livre IV pour les Vierges du Soleil, et du chapitre 16 du même livre pour l'interprétation du nom donné à Yahuar Huaca. Elles sont à la fois chambre d'écho et amplification au texte des Contes péruviens dans la mesure où elles renforcent l'atmosphère exotique par des précisions ou des traductions du quechua; elles servent donc le texte. "Sorte de loi du genre», dit Christelle Bahier-Porte dans son article, "Les notes dans les premiers contes orientaux ${ }^{53}$ ", en parallèle étroit avec la relation de voyage, elles prolongent l'écriture et participent dans cette séquence du «zèle pédagogique » que Christelle Bahier-Porte refuse à Gueullette pour les premiers contes orientaux. 
59 En revanche, l'ouverture de la deuxième séquence instaure un nouveau rythme dans le tissu textuel. Le cadre incaïque est tracé, aussi les rappels historico-mythiques ne se font-ils que sous forme de notes longues d'éclaircissement sans inférer sur le corps même du récit mais rappelant sans cesse la présence active du narrateur.

60 Il peut être intéressant de souligner que l'abondance de notes explicatives est nettement moindre dans les Heures que dans le conte-cadre : le plaisir de conter à la façon des Mille et Une Nuits l'emporte sur le plaisir d'instruire. Elles sont également d'une autre teneur et rejoignent alors l'analyse que fait Christelle Bahier-Porte de la note considérée comme un "surplus " par rapport au texte : «De lecture facultative, elle peut devenir le lieu du commentaire critique, mais aussi des prises de distance ludiques $[. .]$.$» .$

61 C'est dans le récit-cadre que le recours aux chroniques qui relatent la fondation et l'organisation de l'empire inca est constitutif: l'utilisation de ces sources donne autorité au texte des Contes péruviens même si elles ne sont pas directement nommées. Mais les lecteurs du XVIII ${ }^{\mathrm{e}}$ siècle étaient plus aptes que nous à reconnaître la trace des chroniqueurs du XVI ${ }^{\mathrm{e}}$ siècle et peut-être à apprécier la facture orientale issue des Mille et Une Nuits. La technique du conteur est constante dans l'utilisation des sources : il a l'art de choisir les éléments qui, par leur caractère mythique et fabuleux, mais parfois erronés du point de vue historique, participent spontanément de l'écriture du merveilleux spécifique aux contes; il élague et synthétise en ce sens les informations des chroniques qu'il prolonge en inventant des événements et en donnant une épaisseur psychologique aux personnages qu'ils n'avaient pas dans les chroniques; ou bien il n'hésite pas, lorsque la tonalité épique des chroniques lui convient, à en restituer le texte presque littéralement au risque, cette fois-ci, de nuire à la vraisemblance psychologique de son héros. Mais il procède toujours avec une liberté de choix qu'il met au service de sa création littéraire.

62 L'auteur - ou les auteurs? - des Mille et Une Heures, Contes péruviens a bien ré-orienté l'encadrement de son recueil plein-Sud et non à l'Est comme les précédents Mille et $U n[e]$, afin de répondre à l'attrait scientifique pour l'Amérique dite latine ; attrait qui coïncidait avec l'actualité des grands voyages de découverte au moment où Voltaire pesait de toute son influence dans la préparation de l'expédition de La Condamine. Les Contes péruviens traduisent à leur manière l'impact de ce projet et le conte-cadre se rapproche de la littérature de voyage tant prisée par les esprits éclairés du XVIII ${ }^{\mathrm{e}}$ siècle.

Répondant à l'horizon d'attente d'un lectorat déjà fidélisé, répondant au goût du temps et des salons, reprenant sans doute matière et ingrédients dont les auteurs de contes orientaux avaient usé auparavant, l'auteur n'en fait pas moins œuvre originale de deux manières : nous ne trouvons en aucune façon un copier-coller servile de la chronique aussi historique que mythique de Garcilaso el Inca, mais un modelage créatif et personnel, dans une réécriture des Comentarios reales. Le conteur opère un découpagemontage original, il s'émancipe avec aisance de la trame rigide et relativement répétitive des Commentaires royaux en procédant à des ponctions sélectives dans la matière brute de la chronique, ponctions qui servent le fil directeur de son conte-cadre en sautant du livre I au IV puis au livre V (histoire de Yahuar Huacac) pour revenir au livre I (description de la maison des Vierges choisies et utilisation du poison). Cette stratégie dans l'appropriation de la chronique lui permet de donner vie à ces Incas, à ces Femmes choisies, vierges du culte solaire, sans nulle simplification symbolique. Il façonne des personnages qui, entre patron pré-construit et création fictionnelle, entre 
mentir-vrai et vraisemblance, entre évolution et transformation, acquièrent une épaisseur psychologique au point de devenir une source dynamique de contes enchâssés qui confèrent à la tactique d'écriture une autre dimension que la simple application de la formule classique « plaire et instruire ».

\section{NOTES}

1. Ce codicille se trouve au Minutier central des Archives nationales, joint au testament de T.S. Gueullette dans le dossier du notaire Jean Vivien, à la date du 23 décembre 1766 (cote IX 737).

2. Les Mille et Une Heures, Contes péruviens (tomes I et II), Londres et Paris, chez Nyon, 1759.

3. Voir J.E. Gueullette, Thomas-Simon Gueullette, un magistrat du XVIII ${ }^{e}$ siècle, Paris, 1938, p. 50-54.

4. A.D. Coderre, L'Euvre romanesque de T.S. Gueullette, Thèse de Doctorat, 1934, p. 18. Coderre s'inspirant de la démonstration de J.E. Gueullette qui s'appuie sur l'auteur de la notice du Cabinet des Fées envisage les choses dans un autre ordre (p. 68-70) : il

rapproche Zadig des Soirées bretonnes en concluant: « Nous voyons que les idées de ces deux passages sont identiques : ainsi Voltaire s'est, sans aucun doute, inspiré des Soirées Bretonnes ». En réalité la source est bien antérieure : voir Raymonde Robert, Le Conte de fées littéraire en France de la fin $d u$ XVII à la fin du XVIII siècle, Paris, Champion, 2002 , p. 59 et suiv.

5. Ibid, p. 95-96.

6. A.D. Coderre, ibid, p. 93-96 : sont comparés le passage des déductions de Zadig décrivant le chien de la reine et le cheval du roi et un passage des Soirées bretonnes (éd. de 1712, p. 311 ; rééd. en 1786, Cabinet des Fées) ; J.E. Gueullette souligne leur ressemblance, mais aussi que : « des épisodes analogues se trouvent dans plusieurs contes orientaux notamment dans Peregrinaggio di tre giovanni figliaoli del re Serendippo ", traduction du persan publiée à Venise en 1557 qui peut être la source du conte de Gueullette. Filiation orientale donc ; mais il est intéressant pour saisir l'influence de Gueullette à son époque de noter le lien que l'auteur de la notice a établi entre deux conteurs d'origine et de fortune diverses. Voir R. Robert, ibid.

7. Irène Passeron, À la recherche de la forme de la terre, Paris, CNRS, UMR 48, Centre Alexandre Koyré, mars 1998, p. 6.

8. Hélène Minguet, Introduction et notes au Voyage sur l'Amazone, Paris, La Découverte, 1993, p. 13.

9. Contes péruviens, $\mathrm{p}$. X.

10. A.D. Coderre présente Les Nuits parisiennes comme la première brochure écrite par T. S. Gueullette ; elles constituent : « ... un mélange littéraire de divertissement spirituel, de bons mots, de proverbes, de points d'Histoire et de faits curieux », p. 168. 11. Il est étonnant de constater que les critiques passés de l'œuvre de Gueullette - J.E. et Ch. Gueullette et A.D. Coderre - ont totalement ignoré cette originalité des Mille et Une Heures. Alors que A.D. Coderre analyse brièvement (p.17) l'engouement du public 
du XviIIe siècle pour l'Orient à partir de la traduction de Galland des Mille et Une Nuits (1704-13), il tait le choix de l'auteur des Mille et Une Heures de les situer dans un Nouveau monde précolombien, dans un Pérou inca.

12. Gueullette est un bibliophile éclairé possédant selon A.D. Coderre une « magnifique bibliothèque » (ouvr. cité, p. 17, 26, 37); c'est un esprit méthodique et un grand travailleur ; on peut supposer qu'il ait connu les récits de certains chroniqueurs espagnols ; peut-être même le compte rendu de l'expédition de La Condamine, très en vogue dans les salons.

13. J. Baudoin, Histoire des Yncas, Roys du Perou, Paris, A. Courbé, 1633.

14. T.F. Dalibard, Histoire des Incas, rois du Pérou, nouvellement traduite de l'espagnol Garcillasso de La Vega, et mise dans un meilleur ordre; avec des notes et des additions sur l'histoire naturelle de ce pays, Paris, Prault fils, 1744.

15. Éditorial Mercurio de Lima, dans son édition de 1998, (qui est celle dont nous nous servons) signale, dans le prologue, une vingtaine de traductions françaises depuis le dix-septième siècle : l'intérêt pour ce texte n'est donc pas éteint.

16. T.F. Dalibard, ouvr. cité, « Préface du traducteur », p. IX.

17. Ibid, p. VIII.

18. Editorial Mercurio, ouvr. cité, t. II, p. 6

19. Raimundo Lazo, Historia de la Literatura Hispanoamericana, la Habana, Editora Universitaria, 1998, p. 135 à 146.

20. Bernard Lavallé, L'Amérique espagnole de Colomb à Bolivar, Paris, Belin, 1993, p. 212. 21. Alfred Metraux, Les Incas, Paris, Seuil, 1961. Ethnologue américaniste, fondateur et directeur de l'Institut d'Ethnologie de Tucuman, homme de terrain autant que théoricien, il était connu pour sa rigueur scientifique. Claude Lévi-Strauss, commentait ainsi la publication des Incas, dans la revue française d'anthropologie, L'Homme (1962) : «Il n'existe certainement pas [...] de meilleure introduction à la connaissance des sociétés péruviennes, [...] les américanistes eux-mêmes y trouveront beaucoup de renseignements naguère épars, ici rassemblés pour la première fois [...] ». Métraux disait des Commentaires: « Aucune monographie moderne ne saurait dispenser [...] de lire les Commentaires royaux. S'il est vrai que cette chronique, écrite au début du XVII ${ }^{e}$ siècle par le fils d'un conquistador et d'une princesse inca à la gloire de ses ancêtres maternels, ne jouit plus aujourd'hui d'un crédit aussi total que par le passé, elle reste cependant inégalée par le charme du style et la richesse de l'information. » p. 185. 22. Voir l'analyse du récit-cadre dans ce recueil par Jean-François Perrin : « Les transformations du conte-cadre des Mille et Une Nuits dans le conte orientalisant français du début du XVIII ${ }^{\mathrm{e}}$ siècle ", RHLF, $\mathrm{n}^{\circ}$ 4, 2004, p. 54.

23. A. Métraux confirme en partie la note explicative du conteur, insistant sur la curiosité allochtone : « Aucune institution incaïque n'a excité autant de curiosité que celles des Vierges du Soleil, dont le nom quechua aclla-cuna, signifie simplement « les femmes choisies ». Elles se recrutaient parmi les jeunes filles que des fonctionnaires spéciaux choisissaient dans chaque communauté et qu'on enfermait dans certains établissements jusqu'au moment où l'Inca ou son représentant décidaient de leur sort. Celles qui ne devenaient pas des concubines de l'empereur ou qui n'étaient pas distribuées aux hauts fonctionnaires ou réservées pour les sacrifices humains étaient rattachées à un temple. Elles y préparaient les aliments cérémoniels, en particulier la chicha (bière de maïs) [...]. Elles formaient de véritables ateliers d'où sortaient des tissus particulièrement réputés [...]. Chaque couvent d'aclla-cuna était placé sous 
l'autorité d'une femme considérée comme l'épouse du Dieu solaire. Le plus grand et le plus célébré, celui du Cusco, comprenait près de quinze cents femmes ».

24. Schahriar renoncera à l'exil s'il rencontre plus malheureux que lui en amour. 25. Quelques chroniques : Pedro Cieza de León, El señorío de los Incas, 1550 ; Juan de Betanzos, Suma y narración de los Incas, 1551 ; Diego de Ortega y Morejón, Fray Cristobal de Castro, Relación de Chincha, 1558. Cette dernière relation est une source précieuse, leurs auteurs décrivant avec précision le système de domination inca sur la côte centrale : administration, système d'impôts, rigueur des châtiments... Elle permet en outre d'apprécier comment, à partir d'un " protectorat » basé sur les échanges commerciaux, les Incas arrivaient à étendre leur domination absolue.

Blas Valera - Historia de los Incas, 1585 - est un chroniqueur métis, prêtre ; il reçut pour mission de compiler tout ce qui avait trait à l'époque inca et voyagea jusqu'à Cadix pour superviser l'impression de son ouvrage; mais par la faute du sac de la ville par les Anglais, il ne resta que quelques chapitres de son Histoire, qu'il remit entre les mains de Garcilaso avant de mourir. Garcilaso s'appuie d'ailleurs en maints endroits de son œuvre sur les assertions du Père Valera ; Anello Oliva : Historia del Reino y Provincias del Perú y Vida de los varones insignes de la Compañía de Jesus, 1631.

26. Garcilaso de La Vega, ouvr. cité, chap. 15, livre I, p. 35.

27. J. Baudoin, ouvr. cité, p. 71-72.

28. Garcilaso, ouvr. cité, $p$. 36 et suiv.

29. J. Baudoin, ouvr. cité, livre I, p. 72 et suiv.

30. Garcilaso, ouvr. cité, livre I, chap. 18, p. 40 et suiv, et J. Baudoin, ouvr. cité, p. 84-87.

31. Voir, entre autres, le chapitre 21 , livre $\mathrm{I}:$ « Enseignements que l'Inca donna à ses vassaux $"$.

32. Voir chez Garcilaso, les chapitres 13 et 14 du livre II, tome I, p. 86-91 : « De quelques lois que Les Incas appliquèrent $»$.

33. Contes péruviens, ouvr. cité, p. 3-4.

34. Garcilaso, ouvr. cité, t. 1, livre II, chap. 6, p. 72 : nous traduisons nous-même le texte des Comentarios reales.

35. Garcilaso, au chapitre 1, livre VII, tome III, p. 7-10 explique le rôle unificateur des langues, en particulier du quecha, dans la colonisation : « Los Incas hacían colonias. Tuvieron dos lenguajes : Les Incas colonisaient. Ils eurent deux langues».

36. Garcilaso, chap. 19, livre I, tome I, p. 43 : « Protestations de l'auteur sur l'histoire » : « ... maintenant que nous avons posé la première pierre - quoique fabuleuse / mythique /- de notre édifice sur l'origine des Incas. » ("... y a que hemos puesto la primera piedra, aunque fabulosa, de nuestro edificio »).

37. La majorité des chroniqueurs est d'accord sur le nombre et la descendance des Incas. Bien que l'histoire de Viracocha se confonde parfois avec celle de son fils Pachacutec, on commence à avoir, à partir de son règne, des éléments historiquement contrôlables. Viracocha a vécu autour de 1430, il organisa les entrepôts de l'Inca dans les villages, déclara la langue quechua " langue officielle », construisit des routes et lança les système de ceques, sortes de bornes signalant les distances.

38. Cristobal Vaca de Castro, premier gouverneur du Pérou, remplaça Francisco Pizarro à la mort de celui-ci. Il fut chargé de rechercher les causes de la guerre civile entre Espagnols et de réorganiser le territoire conquis. Il s'opposa à Diego Almagro qui s'était emparé du pouvoir à la mort de Pizarro. Il recueillit les premiers « témoignages » sur la tradition orale de la généalogie des Incas et sur leurs conquêtes. Il fut membre du prestigieux Consejo de Indias de 1557 à 1561. 
39. Contes péruviens, ouvr. cité, p. 20-26.

40. Garcilaso, ouvr. cité, livre IV, chap. 20, p. 39.

41. Ibid, livre IV, chap. 3, p. 12 ou J. Baudoin, p. 417-418.

42. Garcilaso, ouvr. cité, livre IV, chap. 23, p. 43.

43. Ibid, livre V, chap. 18, p. 82.

44. Contes péruviens, ouvr. cité, p. 46-47 et note de bas de page.

45. Catherine Langle, "Conduire son rêve, la pédagogie du Merveilleux dans Les

Sultanes de Guzarate », Féeries, n 1, Grenoble, Ellug, 2003, p. 107-124.

46. Garcilaso, ouvr. cité, p. 85.

47. Ibid, livre V, chap. 20 , p. 88 ; Contes péruviens, ouvr. cité, p. 56

48. A. Métraux, ouvr. cité, p. 71

49. Garcilaso, ouvr. cité, livre V, chap. 20, p. 89.

50. Contes péruviens, ouvr. cité, p. 58-59 et 99-104.

51. Erreur dans l'exagération : on sait maintenant que les toits étaient de palmes pour laisser passer précisément les rayons du soleil ou de la lune.

52. Garcilaso, ouvr. cité, chap. 21, livre III, p. 169.

53. Christelle Bahier-Porte, "Les notes dans les premiers contes orientaux », Féeries, $\mathrm{n}^{\circ}$ 2, Grenoble, Ellug, 2004, p. 91-108.

\section{RÉSUMÉS}

Porté par une conjonction d'événements dans l'actualité de ce XVIII ${ }^{\mathrm{e}}$ siècle (attrait des grands voyages de découverte, querelle scientifique entre partisans des astronomes Newton et Cassini provoquant l'expédition de La Condamine vers le Nouveau Monde), T.-S. Gueullette choisit d'installer le récit-cadre des Mille et Une Heures dans un Pérou mythologique, celui de l'Inca Yahuar Huacac et de son fils Viracocha. D'une part, T.-S. Gueullette se coule dans l'air du temps et répond au goût des salons de l'époque pour le conte fabuleux, d'autre part, il s'inspire de la lecture des Commentaires Royaux de Garcilaso de la Vega, el Inca.

T.-S. Gueullette va indubitablement puiser la matière (matière plus épique qu'historique, d'ailleurs) chez Garcilaso, premier prosateur hispano-américain, descendant d'un conquistador espagnol et d'une princesse inca, mais à partir de ces ponctions sélectives dans les Commentaires Royaux, il va disposer un fil directeur propre et modeler données et personnages, leur insufflant vie. De surcroît les ponctions qu'opère Gueullette dans le texte de Garcilaso apparaissent riches d'enseignement pour un lecteur $\mathrm{du} \mathrm{XVIII}^{\mathrm{e}}$ siècle aussi bien sur la religion, le culte du Soleil et les Vierges choisies, que sur la stratégie de conquête et de colonisation des Incas.

A Trip to the roots of les Milles et Une Heures. Induced by a conjonction of eighteenth century events, like the taste for long discovery journeys and the scientific quarrel opposing astronomes supporters of Newton's to those of Cassini's (a controversy that led to La Condamine's expedition towards the New World), T.-S. Gueullette chose to set the adventure story of Les Mille et Une Heures in a mythological Peru, that of th Inca Yahuar Huacac and his son Viracocha. Nevertheless T.-S. Gueullette was also influenced by the taste of literary salons of that time for fabulous tales, as well as by Garcilaso de la Vega's Comentarios Reales.

Undoubtedly, T.-S. Gueullette chose his subject from Garcilaso (even if his story is more epic than 
historical), the first Spanish-American prose-writer, descendant of a Spanish conquistador and an Inca princess. Yet, starting from his different selections from Comentarios Reales, Gueullette will set his own leading thread and theme, shape his own characters and give them life. Furthermore, Gueullette's different selections from Garcilaso's text teach the eighteenth century reader a lot about religion, Sun-worship and chosen Virgins as well as the Incas' conquest and colonization strategies.

\section{AUTEURS}

RÉGINE DAOULAS

Université de La Réunion 